\title{
Medicinal importance and therapeutic potential of senegin in the medicine for the treatment of hepatitis: therapeutic role of superoxide dismutase, glutathione peroxidase and catalase in the liver disorders
}

\author{
Dinesh Kumar Patel
}

Faculty of Health Sciences, Sam Higginbottom University of Agriculture, Technology and Sciences, Payagraj, India

Background: Plants based natural products have been used in the medicine for the treatment of various forms of human disorders. Herbal medicine has been well known in the medicine for their therapeutic benefit and pharmacological activities. Herbal medicines have numerous pharmacological activities due to the presence of different phytoconstituents in the medicinal plants. Methods: Numerous scientific databases have been searched and analyze to know the impact of inflammation in various forms of liver disorders including hepatitis. Medicinal importance and pharmacological activities of senegin in the medicine has been evaluated through literature data analysis of various scientific research works. Numerous literature databases have been searched and needed scientific information has been collected and analyzed in the present investigation. All the collected scientific information has been analyzed for the biological importance of senegin for their beneficial aspects against hepatitis and other liver disorders. Protective role of senegin against various form of hepatitis have been also investigated in the present work through literature data analysis of various scientific research work.

Results: Literature data analysis of various scientific research works revealed the biological importance and therapeutic benefit of senegin in the medicine. From the literature data analysis it was found that senegin has anti-inflammatory activity in the medicine. Further senegin were found to have hypoglycemic activities. Literature database analysis of various scientific research works revealed the biological importance of senegin for the treatment of various form of liver complication including Hepatic inflammation. Molecular study literature databases analysis signified the biological importance of superoxide dismutase, catalase and glutathione peroxidase in the human liver disorders.

Conclusions: Literature data analysis of various scientific research works signified the health beneficial role of senegin in the various form of inflammatory disorders including hepatitis. 